\title{
Cystitis: Symptomatology in Women with Suspected Uncomplicated Urinary Tract Infection
}

\author{
Stefan Heytens, M.D., An De Sutter, M.D., Ph.D., David De Backer, M.D., \\ Gerda Verschraegen, M.D., Ph.D., ${ }^{2}$ and Thierry Christiaens, M.D., Ph.D.'
}

\begin{abstract}
Background: Although cystitis in women is very common in general practice, its evolution in symptoms has not been clearly studied. Qualitative research has pointed to other than the classic symptomatology.

Methods: This was a prospective observational study of the symptomatology at presentation and the evolution of the symptoms in treated women with suspected uncomplicated urinary tract infection (UTI). Women consulting their general practitioner (GP) for dysuria, urgency, or frequency produced a urine sample (for bacteriologic processing) and kept a diary until the end of the symptoms. Exclusion criteria included complaints $>1$ week, fever, vaginal discharge, and known pathology.

Results: Of the 300 asked to participate, $148(49 \%)$ returned the diary. Although none of the patients developed acute pyelonephritis, a substantial number of the women had such complaints as feeling feverish (33\% in culture-positive group, 38\% in culture-negative group), back pains (44\% vs. 56\%), and feeling weak and tired ( $71 \%$ vs. $65 \%$ ). Differences between the culture-positive and culture-negative groups were not statistically significant except for the duration of symptoms, which was shorter in the culture-positive group (4 vs. 6 days). More severe symptoms at inclusion were correlated with a longer duration of these symptoms.

Conclusions: The spectrum of complaints in women with suspected uncomplicated UTI is broad and comprises a number of symptoms usually associated with an upper UTI. The occurrence of these symptoms should not automatically prompt GPs to prescribe broad-spectrum antibiotics. Moreover, the duration of symptoms exceeding the recommended duration of antibiotic therapy does not indicate therapy failure and, thus, the need for changing antibiotic therapy.
\end{abstract}

\section{Introduction}

$\mathbf{P}$ HYSICIANS OFTEN CONSIDER an uncomplicated urinary tract infection (UTI) in otherwise healthy women as a trivial and well-known condition characterized by a limited number of complaints (dysuria, urgency, frequency), which is rapidly resolved with a 3-day treatment. ${ }^{1,2}$ Research based on patients' experiences, however, mentions a broad range of symptoms, ${ }^{3,4}$ and although UTI is the most common infectious disease in adult women, the course of these symptoms is not well documented. Correct information about the symptoms and their average duration is important to reassure the patient and to avoid unnecessary reconsultation, referrals, or changes in treatment, such as a switch to broad-spectrum antibiotics. Furthermore, although a bacterial culture of $>100,000$ colony-forming units (CFU)/mL is generally accepted as defining an infection of the urinary tract, many authors describe the same symptoms in patients with lower levels of bacteriuria and even in culture-negative women. ${ }^{4-7}$ It has even been suggested that antibiotic therapy was effective in culture-negative women. ${ }^{8}$

These conflicting observations demonstrate that much is unclear about the clinical evolution of signs and symptoms in women with suspected cystitis who are receiving antimicrobial treatment. This study explored the clinical course of various symptoms, their duration, and the factors that may influence the duration in patients consulting their general practitioner (GP) with complaints suggestive of an uncomplicated UTI.

\section{Materials and Methods \\ Participating GPs and patients}

Between November 2004 and March 2006, we performed an observational study. Sixty-six GPs in 28 practices in the

${ }^{1}$ Department of General Practice and Primary Health Care, and ${ }^{2}$ Department of Clinical Chemistry, Microbiology and Immunology, Ghent University, Ghent, Belgium. 
region of Ghent were asked to include all female patients $\geq 18$ years of age presenting with complaints of dysuria, urgency, frequency. Patients with symptoms of complicated UTI at presentation (symptoms lasting longer than 7 days, axillary temperature $>38^{\circ} \mathrm{C}$ ) or predisposing factors (pregnancy, known urologic or nephrologic problems, diabetes mellitus, other immunocompromising diseases), patients with recurrent UTI (more than three occurrences in the last year), and patients who had received antibiotics during the past 4 weeks or had obvious gynecologic complaints (abnormal discharge, labial irritation, intermittent vaginal blood loss, and vaginal itch) were excluded.

\section{Data collection}

At the first visit, the GP recorded the patient's age and reason for the encounter. Patients were asked to complete a questionnaire about their personal characteristics and to keep a diary about the evolution of their symptoms. The items in the diary were based on qualitative research performed by Malterud and Baerheim ${ }^{3}$ in 1994 and on a cross-sectional observational study in 2003, exploring the symptoms experienced by women with a lower UTI. ${ }^{3,4}$ All items that occurred in $>1$ in 3 diaries were retained as scoring categories for the diary. Impact on daily life was also registered. Patients were asked to score symptoms daily on a three-step categorical scale of $0=$ absent, $1=$ moderately severe, 2 =very severe.

\section{Statistics}

Data were analyzed using SPSS 12 (Chicago, IL). A comparison of proportions was performed using the chi-square test. Correlation statistics were used to assess the association between culture growth and the severity of symptoms. Survival analysis was used to both describe the duration of symptoms and explore the influence of various factors on this duration. To assess the correlation between the presence of symptoms and the duration of complaints, symptom severity scores were dichotomized.

\section{Ethical approval}

All patients received written information about the study and gave written informed consent. The study was approved by the ethical committee of the University Hospital Ghent.

\section{Results}

\section{Population}

Three hundred women were included, of whom 148 (49\%) returned the diary. Thirteen patients received no therapy and were excluded from our analysis to avoid bias resulting from differences in management. One hundred thirty-five patients received antibiotic therapy (Fig 1). The patients were between 17 and 80 years old, with a mean age of 41.7 years (standard deviation [SD] 17.1). The average duration of the antimicrobial treatment was 4.68 days ( SD 2.16, median 4). None of the participants underwent antibiotic therapy $>10$ days. Nitrofuranes were prescribed most frequently $(72.8 \%), 11 \%$ of the women took fluorochinolone, and 2 women did not take the prescribed antibiotic.

Participating and nonparticipating women had the same distribution of reasons for seeking healthcare. Participating

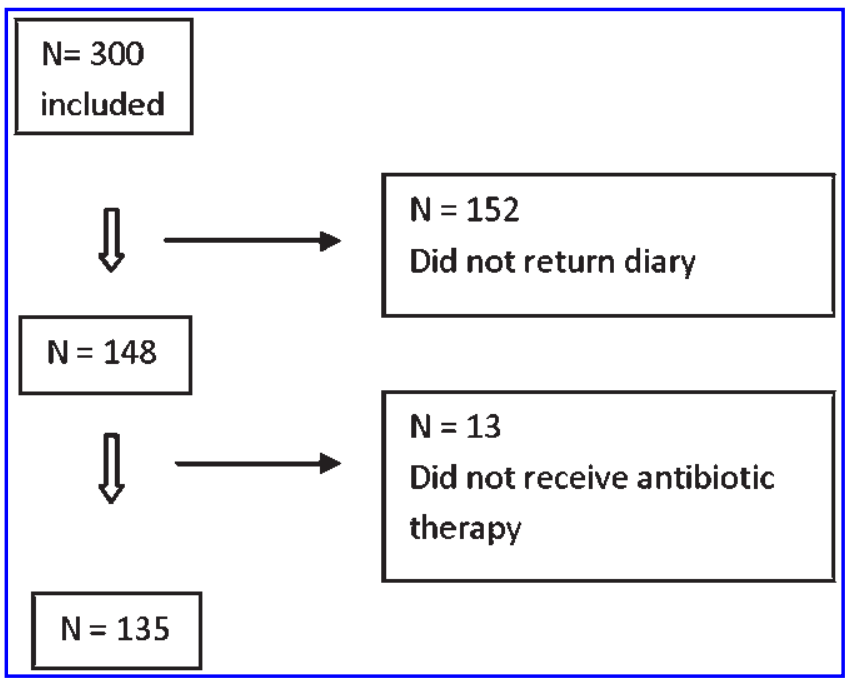

FIG. 1. Flow diagram of included and excluded patients.

women had a higher rate of positive cultures ( $68.7 \%$ vs. $57 \%$, chi-square, $p<0.05)$ and a higher mean age (41.4 years vs. 35.4 years, $p<0.01$ ). As $30 \%$ of our patients had no positive culture growth, we refer to our study population as women with suspected uncomplicated UTI.

\section{Symptoms}

Symptoms were categorized as voiding related, local, or generalized symptoms. ${ }^{4}$ All voiding symptoms, with the exception of incontinence, were highly prevalent, as expected. Apart from the typical voiding symptoms, however, a substantial number of women also had local and generalized symptoms at day 1 (Table 1). Suprapubic pressure, feeling feverish, or feeling weak and tired were very common, al-

Table 1. Frequency of Each Symptom ON DAYS 1, 3, 5, AND 7

\begin{tabular}{|c|c|c|c|c|c|c|}
\hline & \multicolumn{3}{|c|}{ Day 1} & \multirow{2}{*}{$\frac{\text { Day } 3}{\%}$} & \multirow{2}{*}{$\frac{\text { Day } 5}{\%}$} & \multirow{2}{*}{$\frac{\text { Day } 7}{\%}$} \\
\hline & $\mathrm{n}^{\mathrm{a}}$ & $++^{b}$ & $\%$ & & & \\
\hline \multicolumn{7}{|l|}{ Voiding symptoms } \\
\hline Dysuria & 110 & 103 & 93.6 & 56.7 & 25.0 & 15.7 \\
\hline Continuous need ${ }^{\mathrm{C}}$ & 108 & 98 & 90.7 & 52.4 & 13.0 & 8.0 \\
\hline Frequency & 105 & 90 & 85.7 & 30.7 & 6.6 & 10.0 \\
\hline Urge & 106 & 97 & 91.5 & 58.3 & 19.8 & 14.0 \\
\hline Incontinence & 106 & 40 & 37.7 & 20.2 & 4.4 & 2.0 \\
\hline \multicolumn{7}{|l|}{ Local symptoms } \\
\hline Suprapubic pressure & 107 & 80 & 74.8 & 37.9 & 19.6 & 8.2 \\
\hline Pressure in genital area & 107 & 66 & 61.7 & 29.8 & 15.2 & 10.2 \\
\hline Low back pain & 107 & 52 & 48.6 & 27.9 & 15.4 & 14.0 \\
\hline \multicolumn{7}{|l|}{ General symptoms } \\
\hline Unwell & 107 & 57 & 53.3 & 14.4 & 2.2 & 2.0 \\
\hline Weak and tired & 107 & 74 & 69.2 & 33.7 & 14.3 & 12.2 \\
\hline Irritable and restless & 106 & 51 & 48.1 & 18.3 & 5.5 & 4.1 \\
\hline Feverish & 105 & 37 & 35.2 & 7.8 & 5.6 & 0 \\
\hline
\end{tabular}

${ }^{a} \mathrm{n}$, total of patients completing item.

$\mathrm{b}_{+}$, number of patients with symptom.

${ }^{\mathrm{c} C}$ Continuous need, a feeling of a continuous need to void. 
though none of the participants developed an acute pyelonephritis during the 14-day follow-up period.

\section{Bacterial culture and clinical symptoms}

No difference in individual symptoms at presentation was found between culture-positive and culture-negative women (Table 2). These findings were independent of the cutoff value used to define positive culture growth: $10^{5}, 10^{4}$, or $10^{3} \mathrm{CFU} /$ $\mathrm{mL}$. No correlation was found between bacteriuria and the severity of symptom scores.

\section{Duration of symptoms}

The median duration until disappearance of all symptoms was 4 days (95\% Confidence interval [CI] 3-5) (Fig 2). When considering the three symptom categories separately with survival analysis, we found a median duration of 4 days for voiding symptoms, 3 days for local symptoms, and 2 days for generalized symptoms (Fig. 3). This difference in duration was significant (log rank, $p<0.005)$. Women with a positive culture $\left(10^{5} \mathrm{CFU} / \mathrm{mL}\right)$ experienced complaints for a shorter period (median duration: $>10^{5}: 4$ days vs. $<10^{5}: 6$ days; log rank, $p=0.0275$ ). This was also the case when using $10^{3} \mathrm{CFU/}$ $\mathrm{mL}$ as a cutoff level for positive culture growth ${ }^{4}$ (median duration: 4 days vs. 7 days; log rank, $p=0.0380$ ).

There was no significant difference in the duration of symptoms between younger and older women $(<55$ and $>55$ years) (log rank, $p=0.987)$. The duration of symptoms was not related to the length of antibiotic treatment, neither when considering all treatments nor when considering the subgroup of patients taking nitrofuranes (73\%) (log rank: 3-day therapy, $p=0.3452 ; 5$ days, $p=0.2455$; 7 days, $p=0.5259$ ).

A higher severity score of each voiding symptom at first presentation was correlated with a longer duration of the respective symptom (log rank: dysuria, feeling of a continuous need to void, frequency, incontinence, $p<0.005$; urge, $p=0.0413)$. This was also the case for the different categories of symptoms. (log rank: voiding symptoms, $p=0.0034$; local symptoms, $p=0.0065$; generalized symptoms, $p=0.0034)$. Similar results were found for the overall severity score of all symptoms.

\section{Discussion}

\section{Main findings}

The majority of women with suspected uncomplicated UTI experience a number of general symptoms besides the typical voiding symptoms, which is usually considered as indicative of an upper UTI. The median duration of these symptoms is 4 days, which means that $50 \%$ of the patients will experience complaints after finishing antibiotic treatment. ${ }^{1,2} \mathrm{~A}$ higher symptom severity score is correlated with longer duration of the complaints. Finally, positive culture growth predicts a shorter duration of symptoms. No correlation has been found between the extent of bacteriuria at inclusion and the nature of the symptoms and the severity of the symptoms.

\section{A broad range of symptoms}

The literature has previously mentioned that women with uncomplicated UTI suffer from a broad range of symptoms. ${ }^{3,4}$ Our study further substantiates this finding by adding data of a 14-day follow-up period after inclusion. Generalized symptoms, even when present for several days, do not necessarily indicate an upper UTI; they are very common in acute uncomplicated lower UTI. During the follow-up, none of the women with generalized symptoms developed an upper UTI, although most women were treated with nitrofuranes, which are only proposed for use in lower UTIs. Clinicians should be aware that patients feeling unwell, feverish, or weak do not necessarily have a complicated UTI. General symptoms do not differentiate between lower and upper UTI.

\section{Bacterial culture and clinical symptoms}

No correlation was found between bacteriuria and the type of symptoms or symptom scores. Bent et al. ${ }^{9}$ conclude that in

Table 2. Frequency of Symptoms on Day 1 in Negative and Positive Cultures $\left(10^{5}\right)$

\begin{tabular}{|c|c|c|c|c|c|c|c|c|}
\hline & \multicolumn{3}{|c|}{ Positive culture } & \multicolumn{3}{|c|}{ Negative culture } & \multirow{2}{*}{$\begin{array}{c}\text { Difference } \\
\text { in proportions } \%\end{array}$} & \multirow[b]{2}{*}{$\mathrm{p}$ value } \\
\hline & $\%+{ }^{a}$ & $\mathrm{n}+{ }^{\mathrm{b}}$ & $+{ }^{c}$ & $\%+{ }^{a}$ & $n-{ }^{d}$ & $+{ }^{c}$ & & \\
\hline Dysuria & 96 & 75 & 72 & 91.2 & 34 & 31 & 4.8 & 0.373 \\
\hline Continuous need ${ }^{g}$ & 91.8 & 73 & 67 & 88.2 & 34 & 30 & 3.6 & 0.723 \\
\hline Frequency & 84.5 & 71 & 60 & 87.9 & 33 & 29 & -3.4 & 0.770 \\
\hline Urge & 91.5 & 71 & 65 & 94.1 & 34 & 32 & -2.6 & 1.000 \\
\hline Incontinence & 38 & 71 & 27 & 38.2 & 34 & 13 & -0.2 & 1.000 \\
\hline Suprapubic pressure & 72.2 & 72 & 52 & 79.4 & 34 & 27 & -7.2 & 0.483 \\
\hline Pressure in genital area & 59.7 & 72 & 43 & 64.7 & 34 & 22 & -5.0 & 0.674 \\
\hline Low back pain & 44.4 & 71 & 32 & 55.9 & 34 & 19 & -11.5 & 0.303 \\
\hline Unwell & 55.6 & 72 & 40 & 47.1 & 34 & 16 & 8.5 & 0.532 \\
\hline Weak and tired & 70.8 & 72 & 51 & 64.7 & 34 & 22 & 6.1 & 0.654 \\
\hline Irritable and restless & 46.5 & 71 & 33 & 50 & 34 & 17 & -3.5 & 0.835 \\
\hline Feverish & 32.9 & 70 & 23 & 38.2 & 34 & 13 & -5.3 & 0.662 \\
\hline
\end{tabular}

$\mathrm{a} \%+, \%$ of culture-positive women.

${ }^{b} \mathrm{n}+$, number of culture-positive women.

${ }^{c}+$, number with specific complaint.

$\mathrm{d}_{\mathrm{n}}-$, number of culture-negative women.

$\mathrm{e}(\%$ positive culture $)-(\%$ negative culture $)$.

${ }^{\mathrm{f}}$ Fisher's exact test.

${ }^{g}$ Continuous need, a feeling of a continuous need to void. 


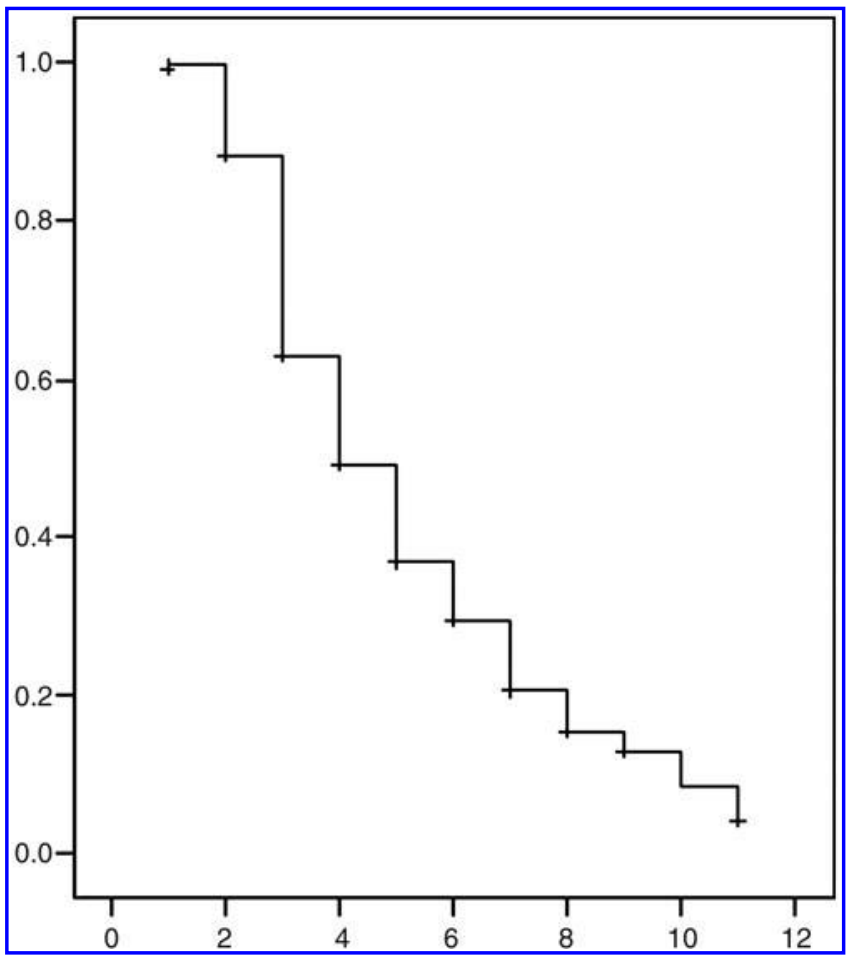

FIG. 2. Duration (days) of all symptoms (voiding, local, and generalized). +, censored.

women with one or more symptoms of a UTI, the probability of an infection is approximately $50 \%$, but that specific combinations of symptoms (dysuria and frequency without vaginal discharge or irritation) increase the probability of UTI substantially (to $90 \%$ ), thus making it possible to base the

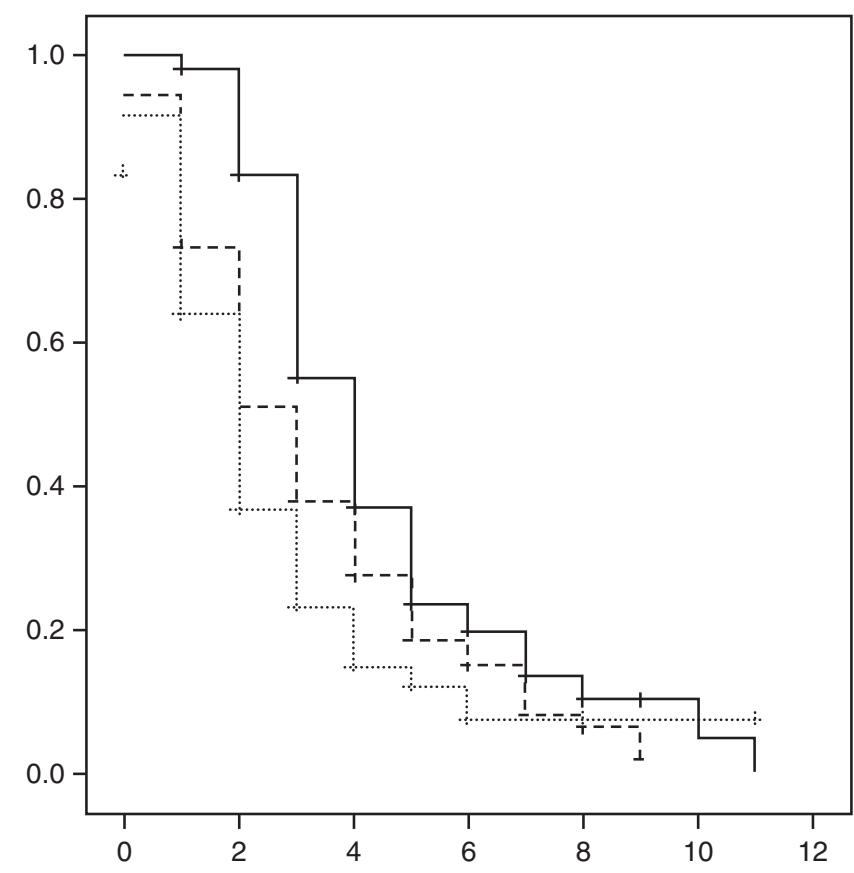

FIG. 3. Duration (days) of voiding (solid line), local (dashed line), and generalized (dotted line) symptoms. +, censored. diagnosis on the history alone. In our study, women with vaginal symptoms were excluded. According to the conclusion of Bent et al., $90 \%$ of the patients included in our study would be expected to have bacteriuria, whereas only $70 \%$ had positive cultures.

Conversely, our results confirm the findings of Ferry et al., ${ }^{10}$ who also found no correlation between symptoms and symptom scores at inclusion, on the one hand, and bacteriuria, bacterial counts, or species on the other. Likewise Baerheim et al. ${ }^{4}$ could not show any differences in frequency or in the pattern of the symptoms between patients with or without bacteriuria. Recently, Little et al. ${ }^{11}$ found only $64.2 \%$ positive bacteriuria in women with suspected UTI, which is very similar to our results.

Nevertheless, GPs are more inclined to treat patients with antibiotics when certain specific symptoms are present, that is, frequency and dysuria or dysuria alone (Fahey et al. ${ }^{12}$ ). As some studies show that these symptoms do not substantially increase the posttest probability of a positive growth culture, a discussion has started in the literature debating the value of individual UTI symptoms for diagnostic testing and treatment in primary care. Our findings support the position that individual symptoms are not very helpful in predicting bacteriuria.

\section{Duration of symptoms}

Overall, the median duration of the complaints was 4 days. This is in agreement with other studies (Ferry et al. ${ }^{13}$ : duration 4 days). Four days after presentation, $50 \%$ of the women still have symptoms. Consequently, clinicians should be careful in promising quick relief of symptoms when prescribing antibiotics. Doctors and patients may be inclined to change or continue the treatment at the end of a 3-day antibiotic course because of ongoing complaints, although this is not an indication of treatment failure.

We found two factors that influenced the duration of symptoms. First, more severe complaints at presentation last longer. To our knowledge, it is the first time that this relation between severity and duration has been investigated, and this may be important to clinicians when informing their patients about the expected course of their complaints. Second, a positive culture is correlated with a shorter duration of symptoms. A possible explanation is that the antibiotic therapy all patients in our study were given may be ineffective in women with symptoms of UTI but who have a negative culture. These correlations were independent of the cutoff for bacterial culture growth used. This finding adds to the ongoing discussion about the most appropriate cutoff value.,

\section{Strengths and limitations}

To our knowledge, this is the first study investigating the correlation between the severity of symptoms or bacteriuria and the duration of symptoms. The design of the diary was based on previous qualitative and cross-sectional research to maximize its validity. ${ }^{3,4}$ A rather high rate of $49 \%$ of eligible patients returned their diaries. The diaries returned were of good quality: almost $75 \%$ of the participating women filled out each item each day.

The participants were on average older and more frequently had a positive bacterial culture. It is unlikely that the age difference caused any bias, as we found no correlation 
between age and duration. The number of patients included was smaller than expected; participating GPs reported that this was mainly due to lack of time and the complexity of the protocol. As the study did not interfere with the treatment, it is unlikely that any bias occurred as a result of selection or exclusion of patients with specific clinical characteristics.

\section{Conclusions}

During a follow-up period of 14 days, the majority of women with suspected uncomplicated UTI symptoms also reported a number of other complaints, apart from the typical voiding symptoms. General symptoms, such as feeling feverish, back pains, and feeling sick-traditionally considered as indicative of an upper UTI-are very common in women with suspected uncomplicated UTI, even when the bacteriologic culture is negative. This is an important finding considering that these symptoms will often prompt GPs to start broad-spectrum antimicrobials, such as fluorochinolones, which may cause unnecessary side effects and the emergence of bacterial resistance. The fact that patients still experience symptoms after finishing treatment does not require modification nor continuation of the treatment. It is interesting for patients who have more severe symptoms at presentation to know that they are likely to experience these complaints for a longer duration, yet it should not lead to a change in therapy.

\section{Acknowledgments}

This work was supported by an unrestricted GSK grant from the Belgian Society for Infectiology and Clinical Microbiology (BVIKM/SBIMC) awarded to D.D.B. There was no contact with the investigators, nor was there influence on the study design and interpretation by GSK. We thank all the participating GPs and patients. Their efforts are much appreciated.

\section{Disclosure Statement}

No competing financial interests exist.

\section{References}

1. Milo G, Katchman E, Paul M, Christiaens T, Baerheim A, Leibovici L. Duration of antibacterial treatment for uncomplicated urinary tract infection in women. Cochrane Database of Systematic Reviews 2005, Issue 2. Art. No.: CD004682. DOI: 10.1002/14651858.CD004682.pub2.

2. Christiaens T, De Meyere M, Verschraegen G, Peersman W, Heytens S, De Maeseneer J. Randomized controlled trial of nitrofurantoin versus placebo in the treatment of uncomplicated urinary tract infection in adult women. $\underline{\mathrm{Br} J \mathrm{Gen}}$ Pract 2002;52:729-734.
3. Malterud K, Baerheim A. Symptom experiences in women with lower urinary tract infection. Scand J Prim Health Care 1999;17:49-53.

4. Baerheim A, Digranes A, Jureen R, Malterud K. Generalized symptoms in adult women with acute uncomplicated lower urinary tract infection: An observational study. Medscape Gen Med 2003;5:1.

5. Colodner R, Eliasberg T, Chazan B, Raz R. Clinical significance of bacteriuria with low colony counts of Enterococcus species. Eur J Clin Microbiol Infect Dis 2006;25:2038-2041.

6. Stamm WE, Counts GW, Running KR, Fihn S, Turck M, Holmes KK. Diagnosis of coliform infection in acutely dysuric women. N Engl J Med 1982;307:463-468.

7. French L, Phelps K, Pothula N R, Mushkbar S. Urinary problems in women. Prim Care Clin office Pract 2009;36:5371.

8. Richards D, Toops L, Chambers S, Fletcher L. Response to antibiotics of women with symptoms of urinary tract infection but negative dipstick urine test results: Double blind randomized controlled trial. BMJ 2005, doi:10.1136/ bmj.38496.452581.8F.

9. Bent S, Nallamothu BK, Simel DL, Fihn SD, Saint S. Does this women have an acute uncomplicated urinary tract infection ? IAMA 2002;287:2701-2710.

10. Ferry SA, Holm SE, Stenlund H, Lundholm R, Monsen TJ. Clinical and bacteriological outcome of different doses and duration of pivmecillinam compared with placebo therapy of uncomplicated lower urinary tract infection in women: The LUTIW project. Scand J Prim Health Care 2007;25:49-57.

11. Little P, Merriman R, Turner S, et al. Presentation, pattern, and natural course of severe symptoms, and role of antibiotics and antibiotic resistance among patients presenting with suspected uncomplicated urinary tract infection in primary care: Observational study. BMJ 2010;340:b5633.

12. Fahey T, Webb E, Montgomery AA, Heyderman RS. Clinical management of urinary tract infection in women: A prospective cohort study. Fam Pract 2003;20:1-6.

13. Ferry SA, Holm SE, Stenlund H, Lundholm R, Monsen TJ. The natural course of uncomplicated lower urinary tract infection in women illustrated by a randomized placebo controlled study. Scand J infect Dis 2004;36:296-301.

Address correspondence to: Stefan Heytens, M.D.

Department of General Practice and Primary Health Care Ghent University

De Pintelaan 185 9000 Ghent Belgium

E-mail: stefan.heytens@ugent.be 
